\section{Aneurysmal Changes at the Basilar Terminus in the Rabbit Elastase Aneurysm Model}

We read the interesting report by Dai et $\mathrm{al}^{1}{ }^{1}$ wherein the authors examined histology at the basilar terminus (BT) following right common carotid artery (RCCA) ligation as a part of a procedure to create an elastase aneurysm model in 30 consecutive New Zealand white rabbits at various time points. The authors concluded that in contrast to our previous report, ${ }^{2}$ there was no bulgelike localized dilation associated with a missing internal elastic lamina (IEL) to suggest microaneurysm or nascent aneurysm formation at the BT, at any time point after RCCA ligation.

We find this report particularly interesting because the first aneurysmal changes at the rabbit BT that we noticed actually occurred in 2 rabbits that had undergone RCCA ligation. ${ }^{3}$ The rabbits underwent the same surgical procedure as that performed by Dai et al, ${ }^{1}$ with the same objective of creating the elastase aneurysm model on the RCCA stump for an endovascular device testing. ${ }^{3}$ We examined whether there were any aneurysmal changes in the basilar bifurcation in these 2 rabbits through histologic staining of contiguous specimens with hematoxylin-eosin (HE), Van Gieson, and trichrome. In the first rabbit, sacrificed 10 weeks after RCCA ligation, we observed loss of IEL, endothelial cells, and smooth muscle cells, a thinned media, and an outward convex bulge at the BT (Fig 1). Fifteen consecutive sections of $15-\mu \mathrm{m}$ thickness consistently presented this BT bulge with missing IEL and media thinning. We repeated the observation in the second rabbit, which had received incomplete RCCA ligation and was sacrificed 12 weeks later. We saw similar aneurysmal changes, including IEL loss and medial thinning at the BT, albeit with a shallower and longer bulge (Fig 2). These vascular defects were clearly aneurysmal and could not be a staining or sectioning artifact, or misinterpreted branches.

These 2 cases prompted us to conduct a prospective study of nascent aneurysmal initiation at the BT induced by common carotid artery (CCA) ligation alone, which resulted in the article by Gao et al. ${ }^{2}$
We performed unilateral or bilateral CCA ligation in otherwise unmanipulated New Zealand white rabbits and prospectively examined aneurysmal changes at the BT 12 weeks later. We observed nascent aneurysm formation and its dose dependence on basilar artery flow increase. Since then, we have shifted our model to perform bilateral CCA ligation only. We have seen prominent IEL loss at the BT in every one of our rabbits, as early as 2 days and 5 days post-CCA ligation. We are in the process of drafting these results.

Our experience with both the elastase model (reported here) and the nascent BT aneurysm model ${ }^{2}$ obviously differs from that of Dai et al. ${ }^{1}$ We are concerned that in their study, Van Gieson staining was done after washing off previous HE staining on the same specimen. The reliability of the de-staining and re-staining technique in detecting IEL loss has not been verified. Reports in prostate cancer specimens show that the reliability of de-staining $\mathrm{HE}$ and re-staining with cytokeratin could be as low as $58 \% .{ }^{4}$ In our experience, more than 50 sections can be taken from 1 BT tissue specimen; thus, adjacent specimens can be used for different stains. Therefore, de-staining and re-staining of the same specimen do not seem necessary and could have confounded the interpretation of their results.

It is not clear whether Dai et $\mathrm{al}^{1}$ created their animal models prospectively to examine the $\mathrm{BT}$ or performed a retrospective analysis on stored specimens that had previous HE staining. In the latter scenario, there could be sectioning bias as well as a need to de-stain and re-stain sections, rather than to use adjacent sections.

Finally, we respectfully disagree with the speculation by Dai et $\mathrm{al}^{1}$ that our group could have misinterpreted branching vessels as aneurysmal bulges in Gao et al. ${ }^{2}$ Branching vessels would have assumed completely different anatomy than what we have demonstrated, withno IEL loss or media thinning. In addition, multiple contiguous sections throughout the entire paraffin-embedded

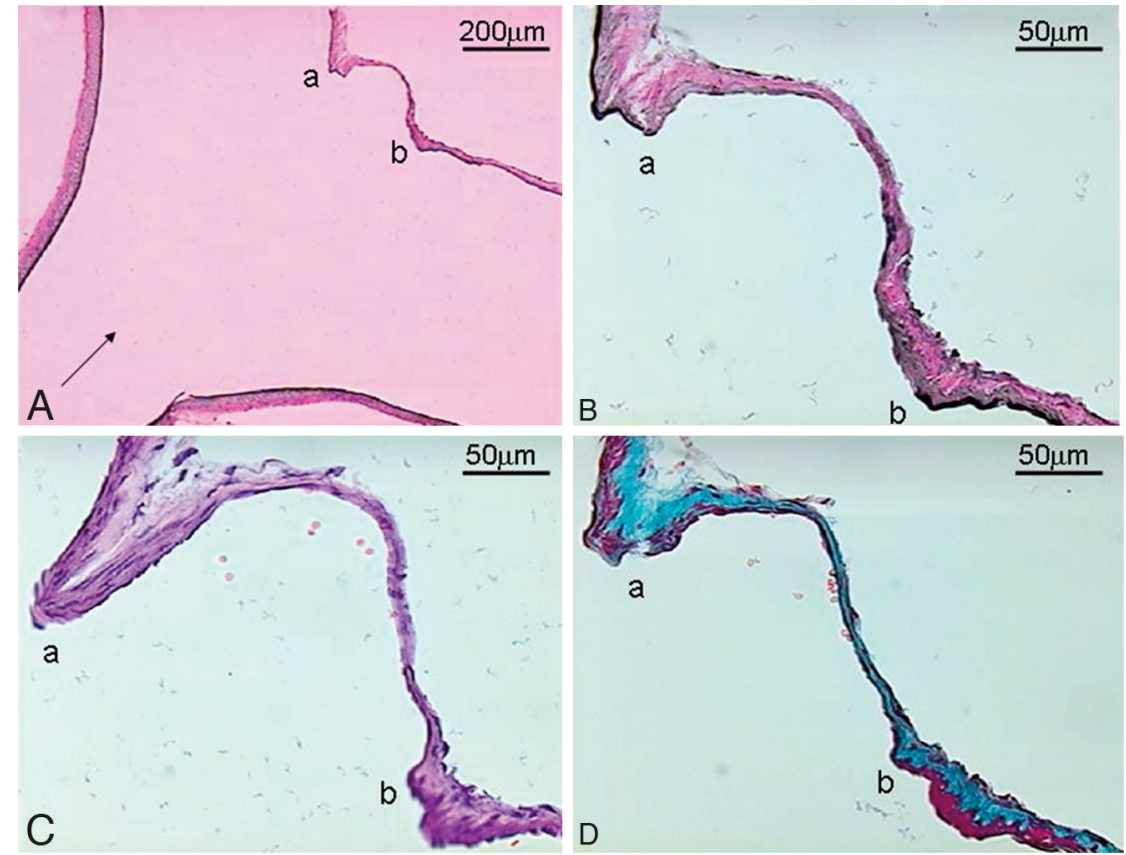

Fig 1. An aneurysm detected at the rabbit BT 10 weeks after RCCA ligation in an elastase aneurysm model. $A$, Basilar artery-posterior cerebral artery bifurcation shown at a low magnification. Arrow indicates blood flow direction. An aneurysm is observed between a and b, with IEL disruption (a total length of $300 \mu \mathrm{m}$ ) and media thinning occurring in the bulge (Van Gieson, original magnification $\times 100$ ). $B-D$, The aneurysm site is shown at a high magnification (original magnification $\times 400$ ). $B$, Van Gieson staining shows IEL Ioss. $C$, HE staining shows endothelial loss. $D$, Trichrome staining shows medial thinning. 


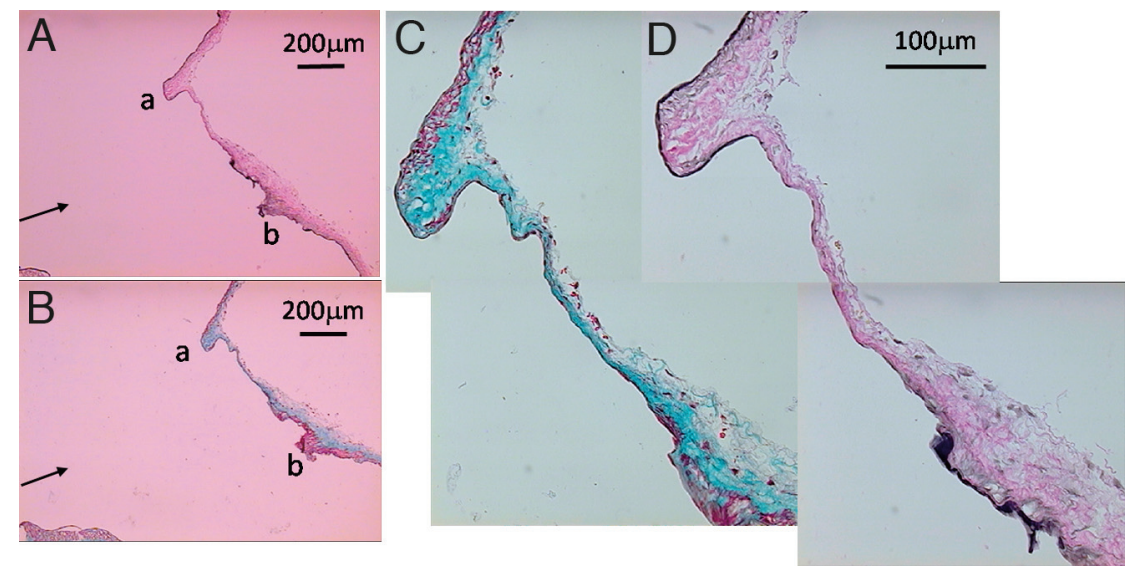

Fig 2. Aneurysmal degradation at the rabbit BT 12 weeks after incomplete RCCA ligation in an elastase aneurysm model. $A$ and $B$, Basilar bifurcation shown at a low magnification (original magnification $\times 100$ ). Arrow indicates blood flow direction. A shallow bulge with a total disruption length of $600 \mu \mathrm{m}$ is observed between a and $\mathrm{b}$. $A$, Van Gieson staining. $B$, Trichrome staining. $C$ and $D$, Aneurysmal degradation is shown at a high magnification (original magnification $\times 400$ ). $C$, Trichrome staining shows medial loss. $D$, Van Gieson staining shows IEL disruption.

bifurcation would have consistently revealed the branching ves-

H. Meng sels, instead of the bulges that spanned multiple sections and taS.K. Natarajan pered off, as seen in our studies.

L. Gao

C. Ionita

J. Kolega

A.H. Siddiqui

\section{References}

1. Dai D, Ding YH, Kadirvel R, et al. Experience with microaneurysm formation at the basilar terminus in the rabbit elastase aneurysm model. AJNR Am J Neuroradiol 2010;31:300-03. Epub 2009 Oct 1

2. Gao L, Hoi Y, Swartz DD, et al. Nascent aneurysm formation at the basilar terminus induced by hemodynamics. Stroke 2008;39:2085-90

3. Ionita $\mathrm{CN}$, Paciorek AM, Dohatcu A, et al. The asymmetric vascular stent: efficacy in a rabbit aneurysm model. Stroke 2009;40:959-65

4. Dardik M, Epstein JI. Efficacy of restaining prostate needle biopsies with highmolecular weight cytokeratin. Hum Pathol 2000;31:1155-61 State University of New York at Buffalo Buffalo, New York J. Mocco Department of Neurosurgery University of Florida Gainesville, Florida 